\title{
Digital Media Content Management System Design and Analysis CHANG Mengxing ${ }^{1,}$, QUAN Xuefeng ${ }^{2}$ \\ ${ }^{1,2}$ Nanyang Medical College, Nanyang 473000, China \\ achangmengxing@126.com
}

Keywords: Digital Media; Content Management; J2EE.

\begin{abstract}
With the rapid development of network technology and digital technology to media content-based digital media industry, the rapid growth worldwide. Thus digital media technology gradually being great concern academia and industry, many countries have developed relevant development strategies and objectives. In China, the digital media industry is regarded as a new economic growth point, a hot investment and development direction. This paper studies the digital media content management system for the object, it sounds, images, videos and other content, using digital technology, its acquisition, production, storage, matching and transcoding form of digital objects, and then relying on the Internet, smart phones and other communication carriers to publish and circulate, and finally reaches the user through a variety of hardware and software end. In this paper, digital media technology and content management system integration, design and implement a complete function, well-structured, high-efficiency digital media content management system.
\end{abstract}

\section{Introduction}

With the rapid development of information technology, multimedia technology continues to accumulate, the traditional Internet-based multimedia technology and its single distribution channel, cannot meet the requirements of the current media audience. New ways mobile phones, Internet TV, a new generation of communications technology, as interactive media content consumption, broadening the digital media distribution channels [1-3]. How to effectively manage the existing digital resources, offers support for the operation of the new media business, and expand resources to lay the foundation for future business, has become a hot topic of current research in academia and industry.

This paper addressed through digital media content management system interoperability and sharing of multimedia resources, design and implementation of a content-based digital media asset management as the core system. The system provides users with a digital media resource archive, organize, and publish the overall solution, it has all kinds of digital content aggregation, storage management, multi-service integrated and diversified content publishing capabilities, users can meet the content of the mass media basic needs of the rescue, management, production, publishing and other content management. Meanwhile, this paper digital media content as the core system, provided technical support for future content value-added services.

\section{Content Management System Requirements Analysis}

Digital media industry is a network technology, digital technology, multimedia technology support, to provide users with digital cultural products and services industry, which covers information services, advertising, communication, communication, electronic entertainment, film, animation, publishing and education many other fields [5]. The emergence of various management systems to meet the basic needs for data management in our industry, users. And some office software, Web page creation software, Web database tools for content creation bear for content management systems play storage, audit and other work. On the other hand, application servers, Web servers, and client devices (such as tablet computers, mobile phones, etc.) are served to deliver content through this channel content in an appropriate form, the content delivered to consumers [6]. 


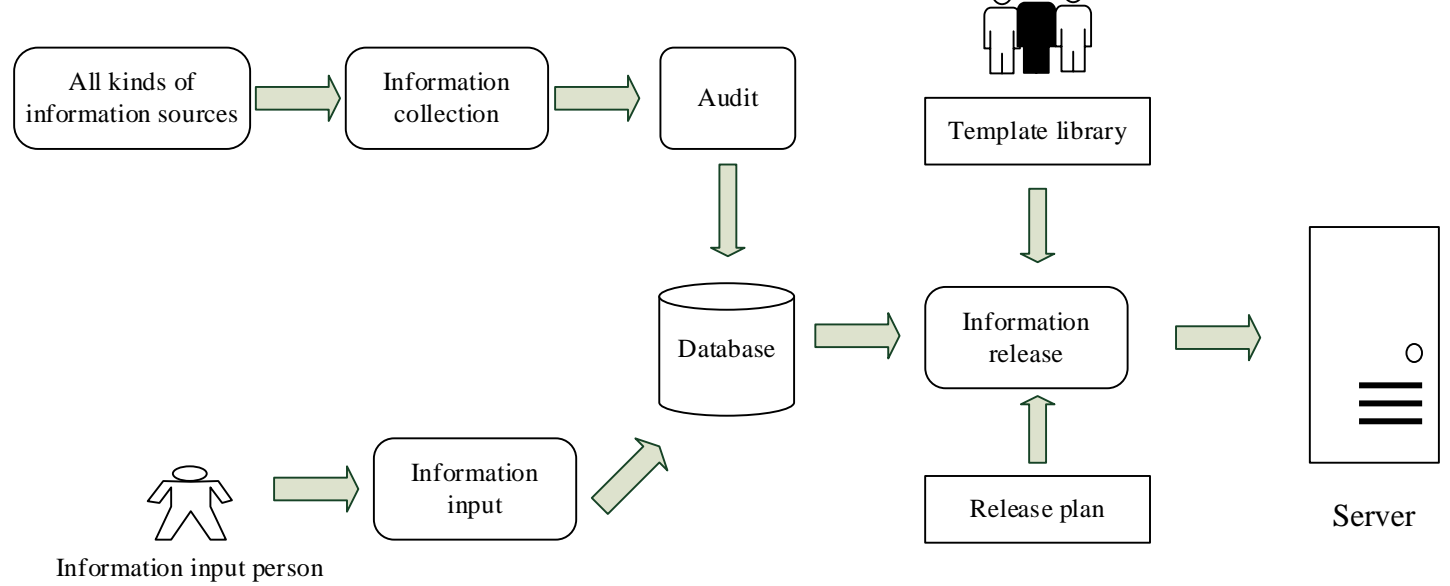

Figure 1. Content management system example

Figure 1 provides an example of a content management processes. Enterprises to accumulate large amounts of current scientific information resources management and development, which is the key to making the right decisions and enhance the competitiveness of the industry; and swept the world in the knowledge economy of today, information for individuals, businesses and society have great value Only for troubles integrate complex information in order to give full play to its value; while enterprises to develop their business, develop new markets, but also an urgent need to manage their content. Content management focus is on a variety of unstructured or semi-structured collection of digital resources, access and delivery, and the characteristics of these resources, to integrate it into the structure of the data management system, so as to these structures of applications to find more extensive data sources.

What kind of content management model can be called good content management? Evaluation of a content management system is efficient and reliable, need to consider whether it can effectively manage different forms, different systems and different servers diversified content resources; whether it needs to effectively manage all forms of information released; whether it is able to businesses and users with a scalable, reliable, secure and cost-effective content of system documentation, multimedia, software products, program code and site structure; it is able to design a variety of content providers content acquisition channels; it whether it can improve the speed of data updates, and content-rich, high service efficiency. At the same time a good content management system can also improve the dispersion of the data from the acquisition, maintenance to the process used to avoid data bottlenecks caused by centralized processing.

\section{Digital media content management system analysis}

The overall structure of the digital media content management system includes the design of a number of modules, including the system registry service module, external interaction module, protocol conversion module, a temporary entity processing module, the basic interface module, service processing module, the process engine processing module, log interface Send event module, component registration services, applications registered service, UI framework interface module. It also includes public DAO, common tools, and business abstraction. The general structure shown in Figure 2. Digital media content management system overall structure of the entire content management system based on J2EE implementation, there is a lot of J2EE design patterns used, the entire content management system also uses a hierarchical design ideas to build the entire system modules. 


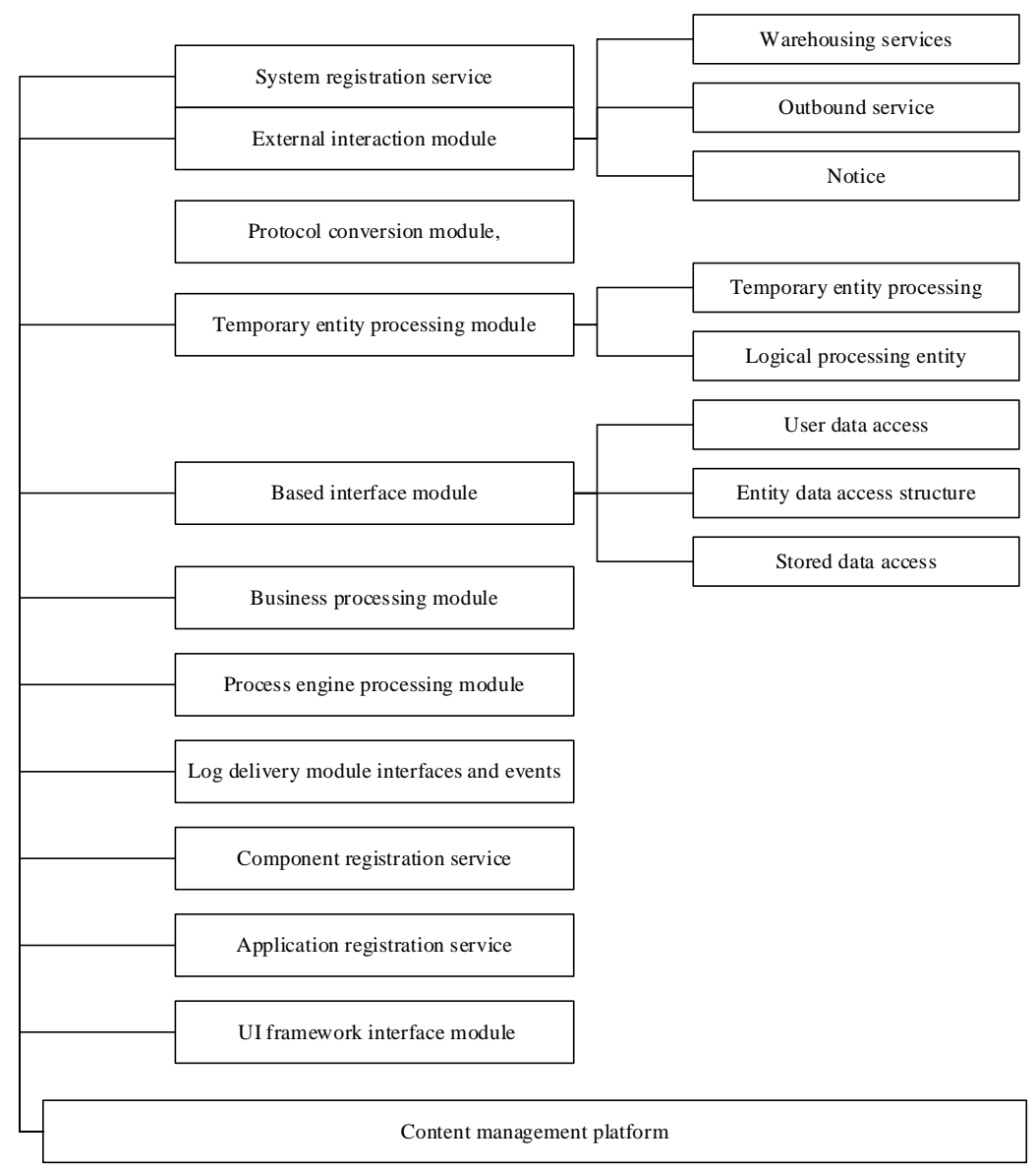

Figure 2. Function module of the digital media content management system

According to the functional division of these modules, the core content management system is the entity, the entity is a content management system for digital content structured description, temporary entity processing module will be temporary entities into entities, temporary entities are handled without internal business entities. Due to the whole system to access the database, the entity persisted to the database, so differentiation out of the base interface module, the database do logic encapsulation, really deal with database is public DAO database underlying operating advanced encapsulation. Content management system center is management content, the external system, content ultimately used by external system, how to need to external systems interact with the system. Through the system registration service, make the system knew external systems. Important features of digital media content management system designed in this paper is to interact with other systems. And this interaction is not completely open interaction.

\section{Design of digital media content management system}

Data is the life of a content management system, in the planning system infrastructure, should hardware, networking, storage, database, operating system and application server that several aspects of the integrated design. Figure 3 is infrastructure design, according to the actual situation of the specific embodiment to be adjusted. 


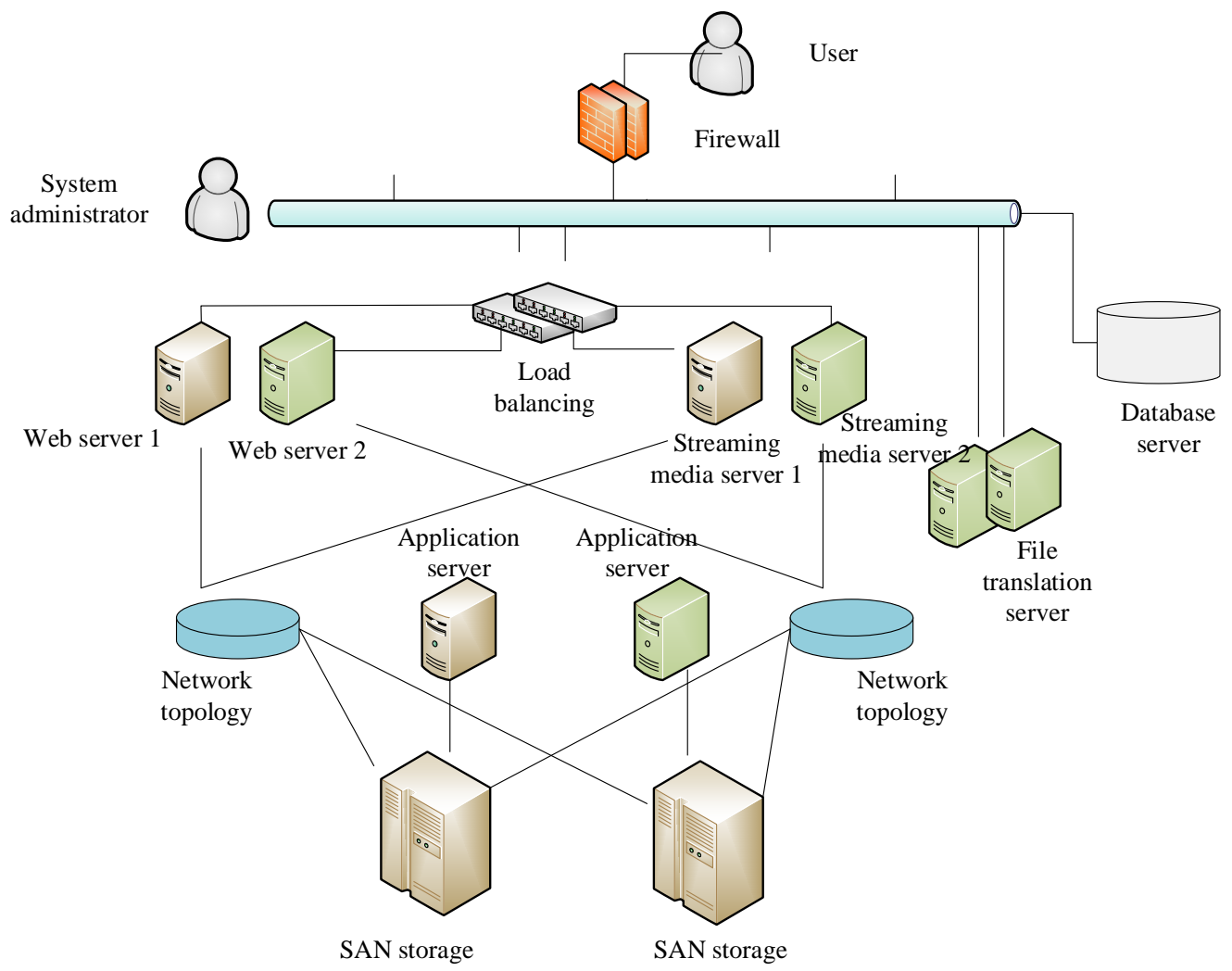

Figure 3. Structure of the digital media content management system

Infrastructure planning content to be from the following several aspects of the design:

(1) Storage system planning. A content management system is significantly characterized by high growth rates and high data storage capacity to store data, so when planning systems storage networks, SAN storage device of choice.

(2) Network planning. With the use of the system, one has to optimize the expansion and upgrade of the problem is the rapid growth of content resources. This article recommends using Fibre Channel switches as the backbone of the network topology, high-performance Fibre Channel switches and Fibre Channel network protocol is key to using SAN storage devices.

(3) Backup system planning. Backup system should not affect the normal operation of the system as a precondition. After the data in the system from unauthorized access, media damage, human error and other causes of loss, a backup system should be able to provide fast data recovery tool. To really ensure the efficient operation of the system and to improve the ability to withstand and recover when disaster occurs, you must make a backup of the data.

(4) Security planning. With the development and growth of the Internet, security has become more important than ever. In order to protect the security of data, network, people have conducted research related to security technology, thus, encryption, authentication, virus protection, intrusion detection, firewall technology emerged. In the information technology infrastructure planning, planning for safety products and technologies are increasingly occupy an important position.

(5) Operating system planning. Operating system after a very long period of development, from a single-user, single-tasking operating system, and the development of a multi-user multi-channel operations and sharing system. OS updates every experienced upgrade multiple versions. When the operating system during the planning, neither conservative nor re blindly.

(6) Database system planning. During the planning aspects of the database, first make sure the data type system is mainly used, the data content, the amount of data, if an existing database, but also the original database software architecture, system architecture, data flow, business, usage system performance through a series of comprehensive analysis and testing. 


\section{Conclusion}

In this paper, the design and implementation of content management system is for digital media. It is the study of digital media, content management to resolve data issues in the field of digital media. The management system is the use of modern digital technology, sound, images and video and other resources for the acquisition, production, storage, matching and transcoding, thus forming a digitized object, then the system is using the Internet, smart phones or other communication carriers for structured or unstructured content publishing and distribution, and finally through to reach the end user a variety of software and hardware equipment. In addition, research on key issues in this article metadata and massive data processing, so that the management system has a broader applicability. In this paper, digital media technology and content management system integration, design and implement a complete function, well-structured, high-efficiency digital media content management system.

\section{References}

[1] Yin H, Liu X, Zhan T, et al. Design and deployment of a hybrid CDN-P2P system for live video streaming: experiences with LiveSky[C]//Proceedings of the 17th ACM international conference on Multimedia. ACM, 2009: 25-34.

[2] Cambria E, Hussain A. Sentic album: content-, concept-, and context-based online personal photo management system[J]. Cognitive Computation, 2012, 4(4): 477-496.

[3] Chen H R, Huang H L. User Acceptance of Mobile Knowledge Management Learning System: Design and Analysis[J]. Educational Technology \& Society, 2010, 13(3): 70-77.

[4] Lonn S, Teasley S D. Saving time or innovating practice: Investigating perceptions and uses of Learning Management Systems[J]. Computers \& Education, 2009, 53(3): 686-694.

[5] Zhang Y, Yu R, Xie S, et al. Home M2M networks: architectures, standards, and QoS improvement[J]. Communications Magazine, IEEE, 2011, 49(4): 44-52.

[6] Fischer G. End-user development and meta-design: Foundations for cultures of participation[M] //End-user development. Springer Berlin Heidelberg, 2009: 3-14. 